\title{
Comparison of polarity in Groningen data with that of other natural and induced seismicity records, and implications in hazard and risk models
}

\author{
İhsan Engin Bal ${ }^{1} \mathbb{D} \cdot$ Eleni Smyrou$^{1} \cdot$ Dimitris Dais $^{1}$
}

Received: 31 May 2018 / Accepted: 8 November 2018

(c) Springer Nature B.V. 2018

\begin{abstract}
Groningen gas field, which is being exploited for more than five decades, has been experiencing shallow and small magnitude earthquakes that cause limited structural damage to the building inventory in the region. These earthquakes are recorded in a relatively small area with multiple recording networks, which constitute a dense strong ground motion sensor grid, providing valuable insight into the characteristics of the motions produced by some $2500+$ small faults at $3 \mathrm{~km}$ depth. The particularities of the Groningen soil, as well as the high seismic vulnerability of the structural inventory, render the Groningen earthquake problem complex, although the magnitudes experienced so far did not exceed $3.6 \mathrm{M}_{\mathrm{L}}$. This paper is an attempt to compare the Groningen earthquake records to records from other induced seismicity and natural seismic events with similar characteristics in terms of magnitude, epicentral distance and depth, for identifying systematic differences in terms of component-to-component variability. In order to achieve that, a total of 1831 recording suites (i.e. couples of horizontal components) are used. 201 of these are Groningen records, 1112 are from other induced seismicity events to geothermal, waste water injection and hydraulic fracturing activities, while 517 are from natural earthquakes. The high polarity of the Groningen records, that is previously reported in the literature and represented as component-to-component variability in risk models, is the main focus of this paper. The component-to-component variability constitutes an important step when implementing ground motion models (GMMs) in risk assessment studies. In this study, in agreement with previous research, the component-to-component variances show that the Groningen induced seismicity events present stronger polarity than the other records used for comparison. The other induced seismicity recordings also show high component-to-component variances as compared to the natural events records, but the main difference is that their variances start decreasing in medium-to-long (i.e. above $0.6 \mathrm{~s}$ ) periods while the variances steadily increase in the case of Groningen records. Furthermore, it is also observed that the component-to-component variances increase considerably when the rotated-to-max-PGA angle, explained in the paper, is used for defining the two horizontal components instead of using components as-recorded or rotated perpendicular to the station back azimuth. A modification to the component-to-component variance model of Groningen GMM v5 has
\end{abstract}

Extended author information available on the last page of the article 
also been proposed in this study for taking into account the orientation of the structural plan directions in respect to the ground motion component directions.

Keywords Induced seismicity $\cdot$ Polarity $\cdot$ Risk models $\cdot$ Strong ground motion

\section{Introduction}

The potential departures of induced seismicity events from the natural events have been checked first by researchers in geoscience disciplines. Research on Basel earthquakes (Goertz-Allmann et al. 2011) showed a relatively low stress drop after the fracture of the fault, an issue which is pointed out also by Sumy et al. (2017). Other similar studies have been conducted for the induced seismicity events in the US. Hough (2014) underlines that a reduction in shaking intensity is expected in case of induced seismicity due to lower stress drop, something that is correlated and at the same time counter-balanced with the generally shallow source depths. Boyd et al. (2017) support that the induced earthquakes are different than the natural ones because the former tend to occur at shallower depths, and in addition are preceded by a relatively large number of foreshocks. Boyd et al. (2017) also suggest that the induced earthquakes at shallow depth exhibit higher ground motion intensities close to the epicentre, i.e. within the first $10 \mathrm{~km}$, as compared to deeper natural events of the same shaking frequency and magnitude. Furthermore, they state that the effect of lower stress drop due to the shallow depth is expected to decrease the high-frequency content of the ground motion within the intermediate field (i.e. from 10 to $100 \mathrm{~km}$ ). Zaliapin and BenZion (2016) defined discriminating signals that can be used to differentiate induced events from the natural ones, but they also underline that there exist catalogue inconsistencies that may obscure such comparisons.

In terms of ground motion models (GMMs), Atkinson (2015) suggests that motions from small and moderate induced earthquakes can be significantly larger than those predicted with the existing models. This fact is related to the shallow focal depths of induced events. In Atkinson and Assatourians (2017), the authors have shown that the ground motion prediction equations by Abrahamson et al. (2014), Atkinson (2015), and Yenier and Atkinson (2015) can be used in functional form and also for overall amplitude level scaling. Yenier et al. (2017) has an important statement for the induced earthquakes in Oklahoma, according to which the commonly drawn conclusion that the induced events have low-stress drops and they produce low ground motion amplitudes is not supported by ground-motion data taken from Oklahoma induced mainshock events at close distances. Wu et al. (2018) report after processing the Oklahoma induced seismicity events that a simple and uniform pattern of stress drop with earthquake magnitude, focal depth, or time was not observed. However, they also state that larger induced earthquakes in Oklahoma definitely tend to have overall higher stress drops. Regarding the intensity of these earthquakes, Atkinson et al. (2018) come to the same conclusions with Hough (2014) in terms of high stress drops, also stating that the natural and Oklahoman induced events exhibit average intensities similar to each other within the first $10 \mathrm{~km}$ to the epicentre. At larger distances, the induced events exhibit lower average intensities as compared to the deeper natural events. 
Bourne et al. (2015) compare 40 Groningen recordings to the GMM presented in Dost et al. (2004) that was predominantly based on Roswinkel gas field in the Netherlands. They conclude that the Groningen data result in significant overestimation. A possible explanation they provide for the large difference in the two fields, both in the Netherlands, is the presence of a high-velocity layer (Zechstein salt formation) immediately above the gas reservoir in the Groningen field. This could act as a barrier reflecting and refracting a large part of the seismic energy. In the Roswinkel field, the Zechstein formation is below the reservoir.

Kaski and Atkinson (2017) compare ground motions from Alberta induced earthquakes, triggered by hydraulic fracturing, oil and gas production, and waste water disposal, to those from Oklahoma, focusing on the empirical definition of response spectral shapes and amplitudes. They conclude that small-magnitude events $(\mathrm{M}<3.6)$ in Alberta have notably lower amplitudes than those in Oklahoma for frequencies above 3-5 Hz, possibly pointing local source effects. They also conclude that the ground motion response spectra from Alberta and Oklahoma induced events are comparable for magnitudes above 3.6.

Finally, Bommer et al. (2017a, b, 2018) discuss the strong polarisation of the two horizontal components as a discriminating aspect of the Groningen records. Namely, there is an unusually high component-to-component variability compared to values obtained from tectonic databases (e.g. Campbell and Bozorgnia 2007). Bommer et al. (2017b) compares the component-to-component variability of suite of Groningen recordings to large suites of tectonic recordings from the European, NGA-West2 and KiK-net databases. They conclude that the $\sigma_{\mathrm{c}}^{2}$ variances presented in Campbell and Bozorgnia (2007) could fit the Groningen data only if multiplied with 2.25. They further develop the GMM (Bommer et al. 2018), and provide a magnitude and distance dependent component-to-component variance model in $\mathrm{v} 5$.

This paper also discusses the possible implications of the high polarity in risk models and suggests a modification to the Groningen-specific component-to-component variance model of GMM v5 (Bommer et al. 2018) for taking into account the orientation of the structural directions in respect to the ground motion components. The issue of orientationdependence of ground motion parameters has been thoroughly discussed by Boore et al. (2006), and a statistical approach has been proposed taking into account the $\mathrm{pp}^{\text {th }}$ percentile of the oscillator responses.

\section{Datasets used for comparison}

This paper compares three datasets, i.e. Groningen induced, other induced and natural records. A comparative dataset has been created for this purpose by using natural seismic events that have similar characteristics in terms of magnitude, epicentral distances and depth. A total of 1831 recording suites (i.e. couples of horizontal components) are used. 202 of these are Groningen records from all 2+ magnitude events available, selected with the condition that at least one of the two horizontal components has a PGA higher than $0.001 \mathrm{~g}$, as values below that would have had no significant effect in any structural terms. 1112 recording couples are from other induced seismicity events in Oklahoma, Alberta, The Geysers, Texas and Switzerland. The Geysers and Switzerland earthquakes are due to geothermal activities, while Oklahoma and Texas induced earthquakes are triggered by waste water injection. Alberta induced earthquakes are reported to be caused by hydraulic fracture or waste water disposal. 517 recording suites come from natural events collected 

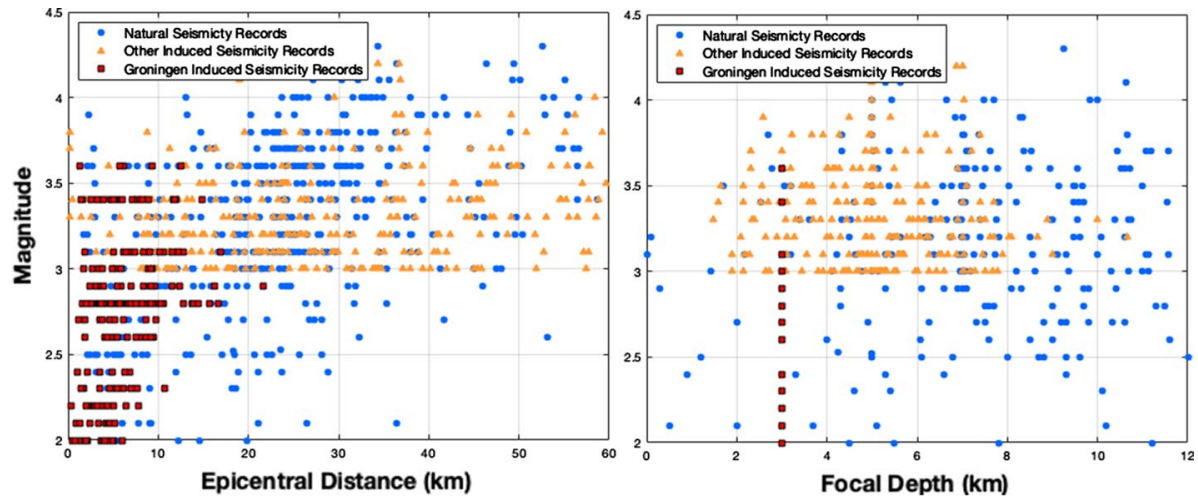

Fig. 1 Comparison of magnitude, epicentral distance and focal depth of the selected records

from US, Italy, Turkey and Greece. Due to the limited magnitude range of the recorded earthquakes, the Groningen records used here come from magnitudes 2.0 to 3.6, while the other induced seismicity and natural seismicity events include larger magnitudes up to 4.5 magnitude. In terms of epicentral distances, the Groningen records used in this work are up to $22 \mathrm{~km}$ epicentral distance while records up to $60 \mathrm{~km}$ epicentral distance have also been included in the dataset from other induced and natural seismicity events. This is due to the difficulty of finding low magnitude shallow earthquakes comparable to the Groningen events. Magnitudes of the selected events, in respect to their epicentral distances and depth, can be seen in Fig. 1.

More details on the data are given in the Data and Resources section of this paper.

\section{Polarity of the horizontal components}

\subsection{Definition of polarity}

One of the particularities the earthquakes in the Groningen gas field exhibit is the strong polarity, the inconsistency between the two perpendicular horizontal components of the same accelerograph set, as explained in Bommer et al. (2017a, 2018) and in Stafford et al. (2018). This inconsistency, manifested as the difference in amplitude between the two perpendicular horizontal components, may be observed in strong ground motion characteristics such as the PGA, PGV, PGD, or in spectral values.

Polarity may be associated with the source and the radiation characteristics in general. The sources of these small induced earthquakes are some 2500+ faults covering the field, mostly oriented in EW and NNW-SSE directions (Bommer et al. 2018). The type of fault ruptures resembles near-point source characteristics (Bommer et al. 2018). The area affected by the induced seismicity earthquakes is relatively small, approximately $78 \mathrm{~km}$ by $44 \mathrm{~km}$, thus the epicentral distances recorded are often smaller than $20 \mathrm{~km}$. The geometric conditions of the earthquakes, such as the high instrument incidence (incidence is the angle from vertical at which an incoming ray arrives, a ray arriving for example from directly below the station would have had an incidence of zero), as well as the short epicentral distances, render the properties of the recorded motions rather complex and thus difficult to compare to other recorded induced or natural earthquakes. Groningen earthquakes occur at $3.0 \mathrm{~km}$ 


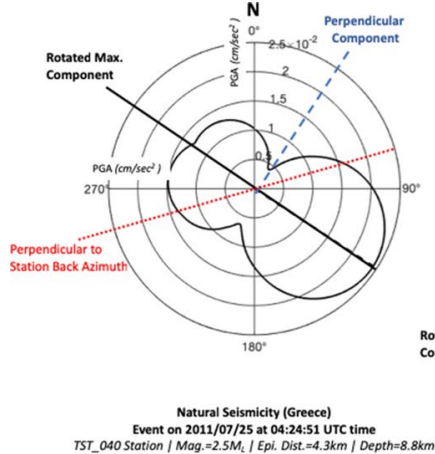

(a)

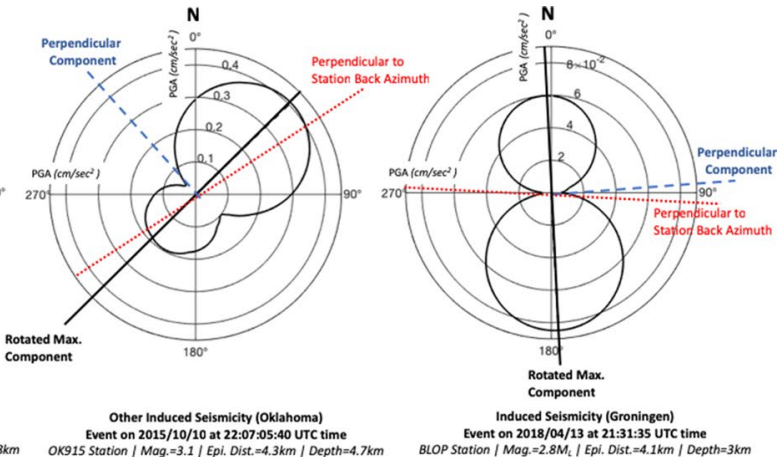

(b)

(c)

Fig. 2 Polarity plots of the records that provide the highest polarity ratio for a natural seismicity, $\mathbf{b}$ other induced seismicity and $\mathbf{c}$ Groningen induced seismicity accelerographs

depth, affecting a soft soil deposit together with Zechstein salt layer above the reservoir and the overlying chalk layer throughout the field. Furthermore, there is a salt layer with giant salt domes inside that $3 \mathrm{~km}$-thickness (Kraaijpoel and Dost 2012) with the potential of diverting or highly affecting the radiation paths and the overall soil deposit response.

The seismic instruments are often placed in an arbitrary direction, thus the horizontal components published in the databases are not related to the travel path or to the faultparallel or fault-normal directions in most cases. Although there are efforts of providing fault-parallel and fault-normal components, the relation of the position of the fault to the instrument may be subjective (Beyer and Bommer 2007). One way of scanning the horizontal components for a principle direction is to rotate both components simultaneously until a maximum is found. For a given $\theta$ angle, this rotation can be done by multiplying the two accelerograms with a rotation matrix as shown in Eq. 1.

$$
\left(\begin{array}{l}
a_{x}^{\prime}(t) \\
a_{y}^{\prime}(t)
\end{array}\right)=\left[\begin{array}{cc}
\cos \theta & \sin \theta \\
-\sin \theta & \cos \theta
\end{array}\right] \cdot\left(\begin{array}{l}
a_{x}(t) \\
a_{y}(t)
\end{array}\right)
$$

Graphical representation of the multiplication explained above is the polarity plot, which is helpful to present the expected PGA values of components if they were placed in a different angle than the as-recorded. Instruments often are placed at EW-NS orientation, with a few degrees of error, as in the case of KNMI network in Groningen. The plots in Fig. 2 show the polarity plot as well as the direction of "rotated-to-max-PGA" (i.e. the component with the highest PGA), the perpendicular component to that, and "perpendicular-to-BAZ" (station back azimuth). It is reminded that the back-azimuth is the angle of the line connecting the station to the epicentre in respect to North in clockwise direction, and the perpendicular direction to the back azimuth is aimed to mark the theoretical shear wave direction that occurs perpendicular to the travel path.

In order to highlight the phenomenon, an example from Groningen is chosen and compared to other examples from natural and other induced seismicity records. The Groningen record from 13th of April 2018 earthquake at BLOP (Loppersum) station is presented in Fig. 2. This record provides the highest polarity among 201 Groningen record couples 

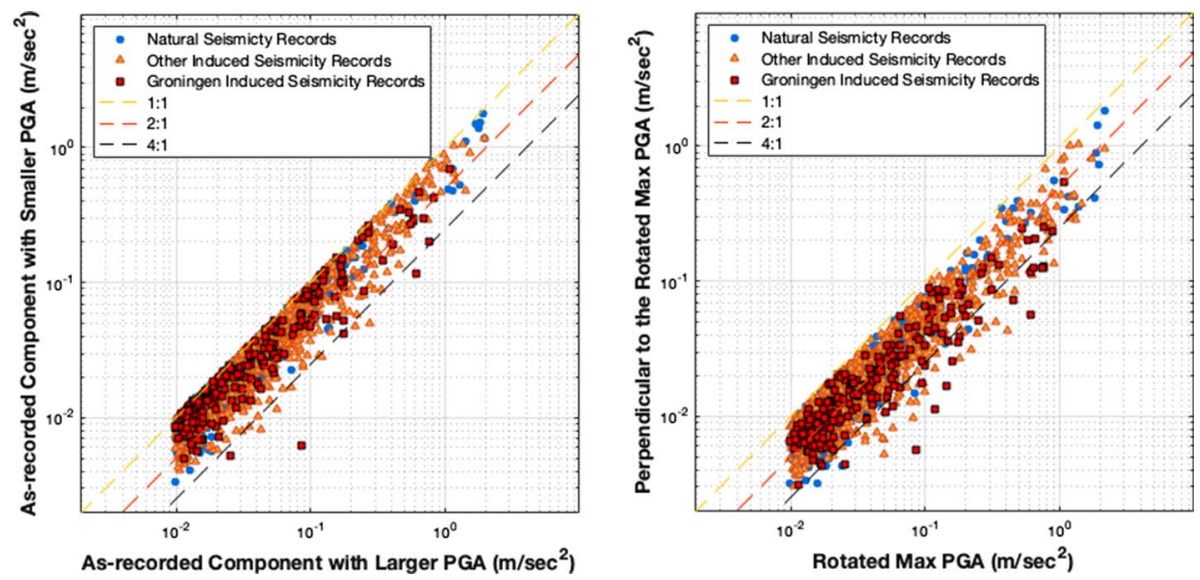

Fig. 3 Comparison of the PGA of the larger arbitrary component to the smaller one (left) and of the strongest component to the perpendicular one (right) for natural, other induced and Groningen induced seismicity events

examined, ${ }^{1}$ both for as-recorded and rotated to maximum PGA directions. The highly pronounced polarity can be clearly seen in Fig. 2 for all records.

\subsection{Comparison of the polarity in Groningen records with others}

The strong polarity was evident in the component-to-component variability in the tailormade GMM models developed for local induced seismicity (Bommer et al. 2018; Stafford et al. 2018). The high polarity exhibited in short distances is expected to have a significant impact on the risk calculations as well. Regional seismic risk analyses are conducted by using simplified models that make use of representative structures such as frames or even single-degree-of-freedom (SDOF) models, the responses of which are monitored in a single horizontal direction. Issues such as the interaction of the plan distribution of stiffness, strength and mass of a building with the two horizontal components of the recorded motions are often ignored or accounted for in an indirect fashion. The GMM spectra, on the other hand, are provided as a sort of combination of the two horizontal components, for example by using geometric mean, and they need to be split into two if analyses in single direction are conducted. The variability of one component in respect to the other plays a role in that splitting process, sometimes creating a much stronger component and a much weaker component than the combined version, if the component-to-component variability is high. One of the particularities of the Groningen houses is the unusually high difference of strength and stiffness in the two perpendicular plan directions (Crowley and Pinho 2017; Crowley et al. 2017). The difference in the two perpendicular components of the seismic demand, which is typically derived

\footnotetext{
1 The highest polarity can in fact be found at BZN1 station data from 8th of January 2018 Zeerijp Earthquake when the complete KNMI database is processed. However, after carefully scrutinizing the data and with the help of Elmer Ruigrok and Bernard Dost from KNMI, it was found out that this recorder did not function properly during the earthquake, thus it has been removed from our dataset.
} 
from the geo-mean GMM spectra, then becomes very much relevant, with the potential of highly influencing the final outcome of the risk study.

Figure 3 shows the comparison of PGA of the component with the maximum PGA to the PGA of the perpendicular component to that. It can be seen that the ratio of the maximum to perpendicular PGA values can go above 4 (up to 14 for Groningen data) for the case of Groningen induced and other induced type of records, when the records are processed as-recorded. Some of the Groningen induced records present ratios of PGA up to 16 when the two components rotated to the direction at which the max PGA value is obtained.

Although Fig. 3 provides a first view of the spread of polarity for the PGA values, a more systematic way, which also includes the spectral accelerations at varying periods, has been followed here. Please note that the rotated spectra are calculated from the rotated time-histories. The evaluation of the component-to-component variability has been done in this paper by using the formula presented by Boore (2005), where the component-to-component variance is defined as the arithmetic mean of the squares of the component-to-component residuals, as shown in Eq. 2. In this equation, $\mathrm{N}$ is the number of records, $\mathrm{Y}_{\mathrm{ij}}$ is the strong ground motion parameter of the ith component of the jth record.

$$
\sigma_{c 2 c}^{2}=\frac{1}{4 N} \sum_{j=1}^{N}\left(\ln Y_{1 j}-\ln Y_{2 j}\right)^{2}
$$

When only smaller $(\mathrm{M}<6)$ earthquakes are considered and recordings are limited to short source-to-site distances, higher component-to-component variances are also found within tectonic databases, but the variability remains smaller than that found for Groningen (Bommer et al. 2017a). The plot in Fig. 4, that consists of natural earthquakes with magnitudes 2-4.3, epicentral distances 2-60 km and focal depths up to $12 \mathrm{~km}$ (blue lines in the figure) supports this statement, while the other induced earthquakes provide high variances, values between those from Groningen and natural datasets.

The Groningen GMM v5 (Bommer et al. 2018) also presents an exercise in which the records are rotated $1^{\circ}$ each time and the component-to-component variability is monitored by rotating the components. The results are summarized in 15-degree intervals, concluding that the difference in variances is not larger than $5 \%$ for median values and not larger than $10 \%$ when maximum values are considered. The outcome of their exercise is reported to be more consistent for the periods below $1 \mathrm{~s}$. An exercise, similar to that, has been conducted in this paper for all three data sets. There is a difference between what was reported in GMM v5 and the plot in Fig. 4, which is the Groningen as-recorded data after $0.6 \mathrm{~s}$ period (see thick red line in Fig. 4). Although the authors could not find a clear explanation for this difference between the Groningen data processed in GMM v5 and the data used in this paper, one explanation can be the type of data used. Groningen GMM v5 (Bommer et al. 2018) makes use of all Groningen data while the dataset in this paper includes only KNMI records from earthquakes above magnitude 2.0, and most importantly, with PGA values above a certain limit $(0.001 \mathrm{~g})$. There are several other available records in the Groningen database, with acceleration amplitudes that are well below the range of interest for structural engineering applications. Such records are also expected to have a low signal-to-noise ratio, a fact that may affect the long-period spectral response, depending on the level of self-noise of the instrument and the filtering strategy followed. 


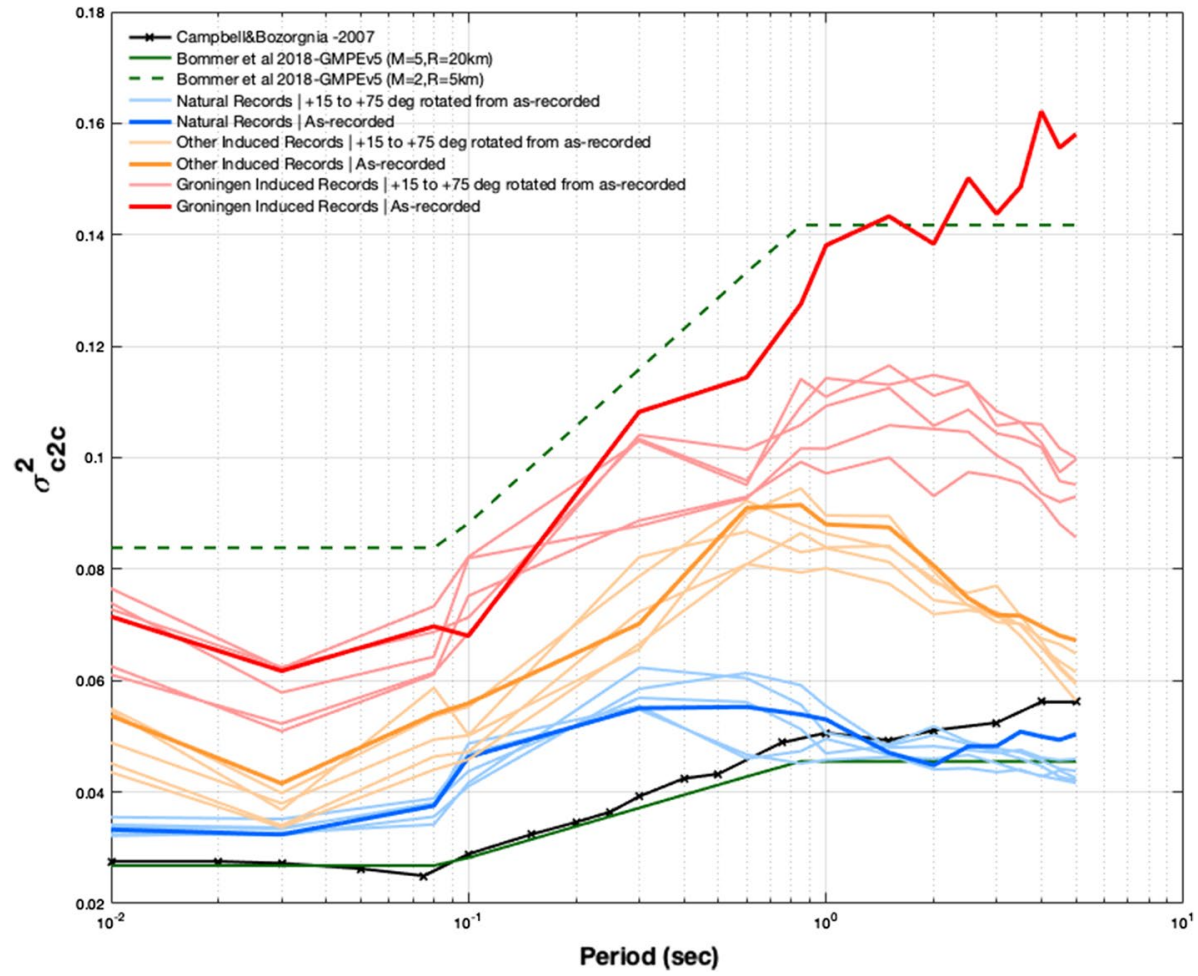

Fig. 4 Comparison of component-to-component variances of Groningen records with tectonic model given by Campbell and Bozorgnia (2007), with Bommer et al. (2018), and with other induced and natural records, where all components have been rotated $15^{\circ}$ at a time

It is rather expected that rotating all stations at the same time, as in the exercise presented in Fig. 4, would exhibit relatively low fluctuations in the component-to-component variance, because this operation is done for all records simultaneously with the same rotation angle. Deviations from component to component are averaged in this way. A different approach is followed in this paper, shown in Fig. 5, to present the sensitivity of the polarity to the instrument angle of instalment. Component-to-component variances are calculated when each record is aligned to the rotated-to-max-PGA direction. The variances between the spectral values of the rotated components are shown in Fig. 5. It can be seen that the already high variances of the Groningen records further increase almost in all period ranges, if each individual record is aligned with the rotated-to-max-PGA direction. Results of as-recorded and rotated-to-max-PGA meet only in very long periods, at around $5 \mathrm{~s}$.

The angle perpendicular-to-BAZ is usually in good correlation with the direction of rotated-to-max-PGA (see Smyrou et al. 2011 for an example from New Zealand). This is also the reason why records are rotated to fault-parallel and fault-normal directions in cases of clear line sources. The correlation of these two angles has been presented in Fig. 6. The mean value of zero in that figure means that the perpendicular-to-BAZ and rotated-to-max-PGA are similar. A plus or minus $\pi$ means that these two angles are in complete opposite directions. It can be seen in Fig. 6 that the median values of all datasets are close to zero, but the standard deviations are relatively high, meaning that 


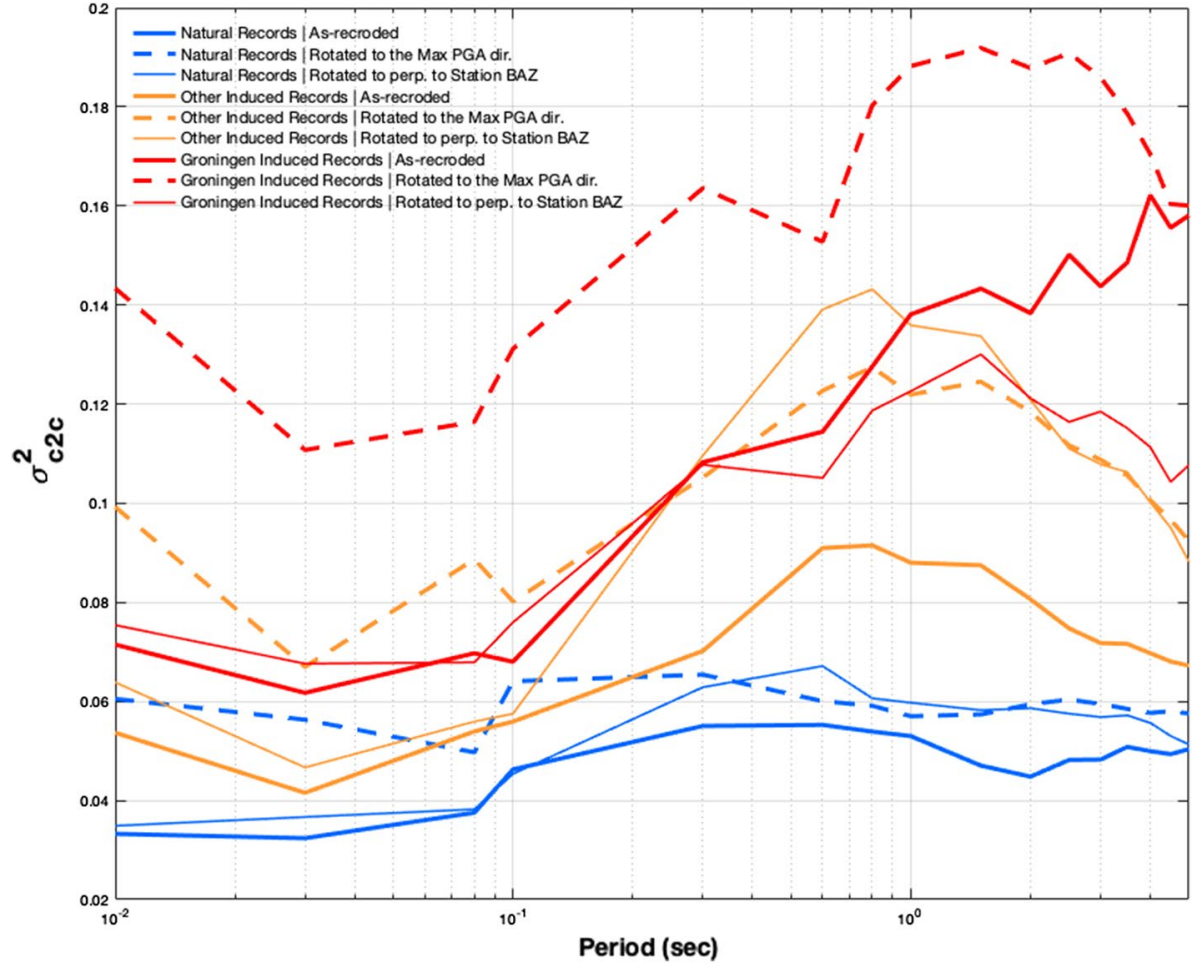

Fig. 5 Comparison of component-to-component variances fort the cases of as-recorded, rotated to the direction of maximum PGA, and perpendicular to the station back azimuth
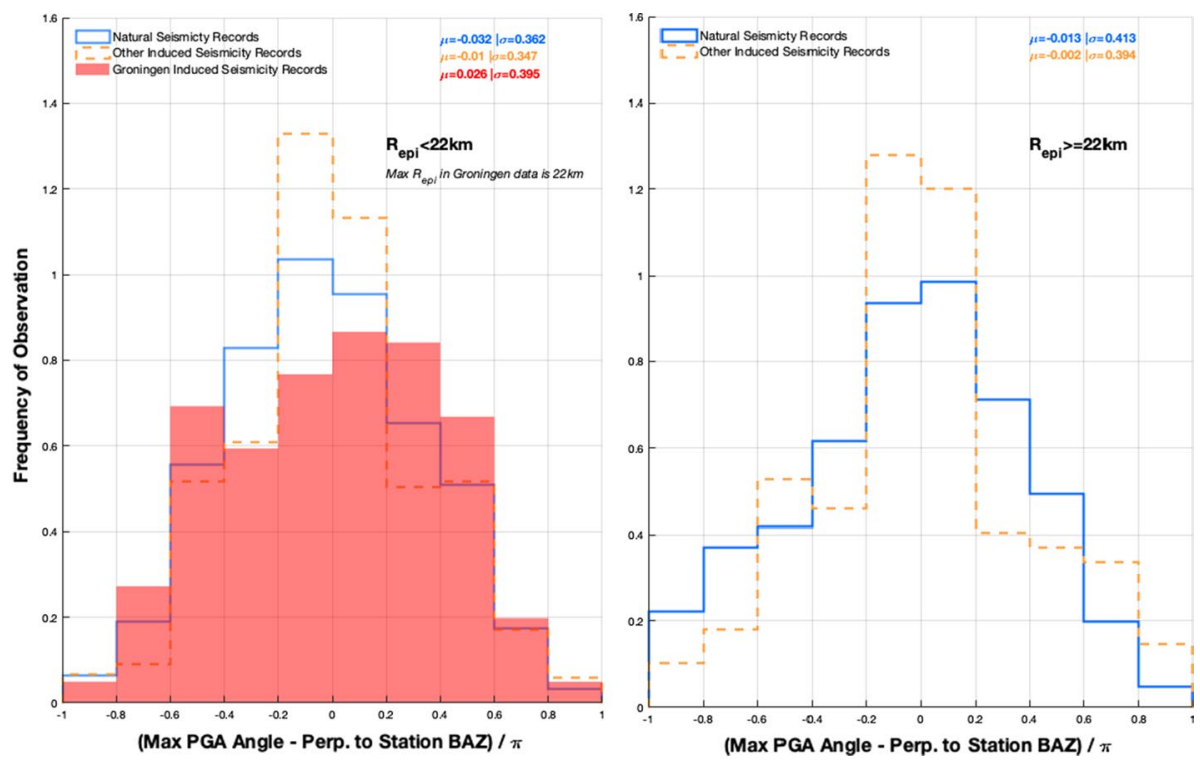

Fig. 6 Distribution of angle difference between the station back azimuth and the direction of the maximum PGA 


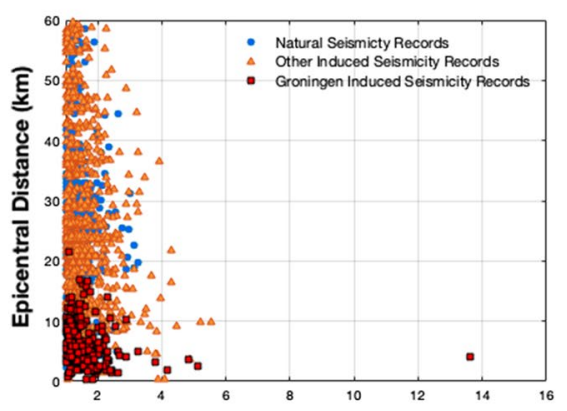

PGA As-recorded Larger Comp. / Smaller Comp.

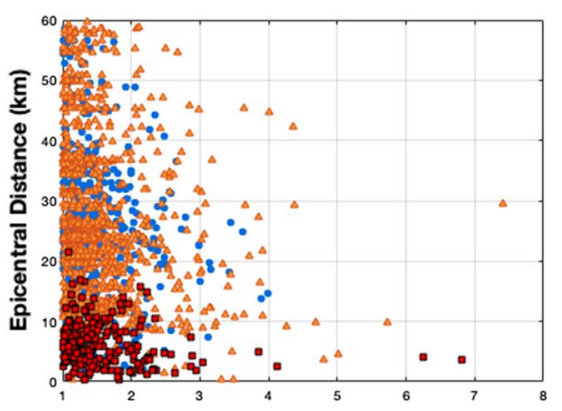

$\mathrm{Sa}(\mathrm{T}=0.1)$ As-recorded Larger Comp. / Smaller Comp.

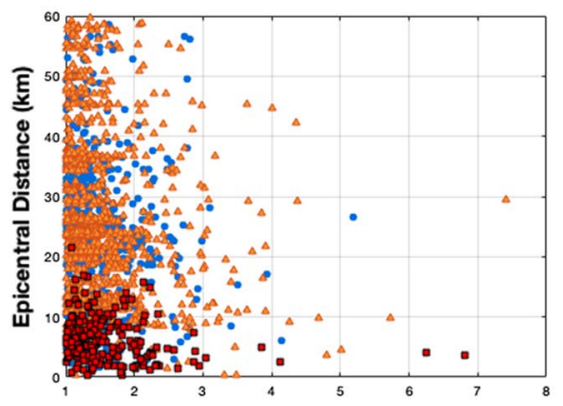

$\mathrm{Sa}(\mathrm{T}=1.0)$ As-recorded Larger Comp. / Smaller Comp.
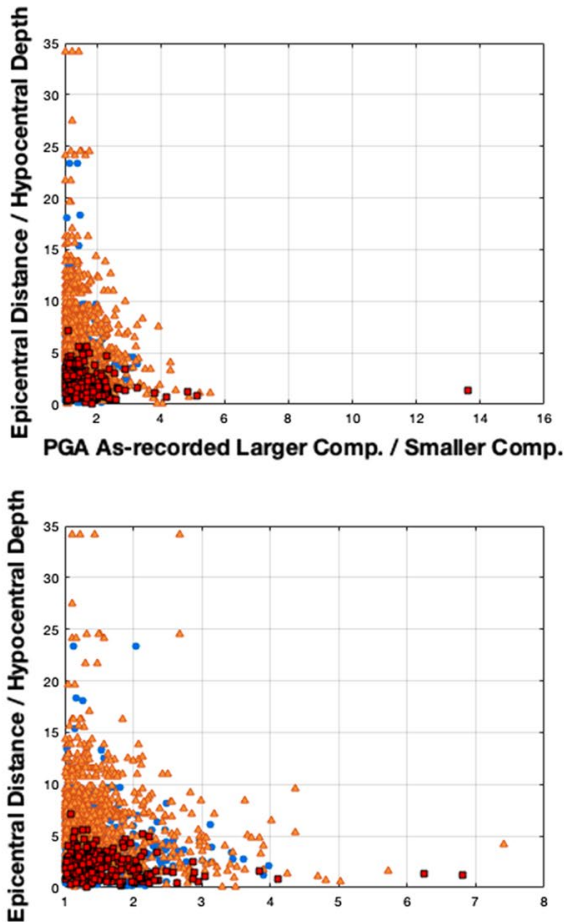

$\mathrm{Sa}(\mathrm{T}=0.1)$ As-recorded Larger Comp. / Smaller Comp.

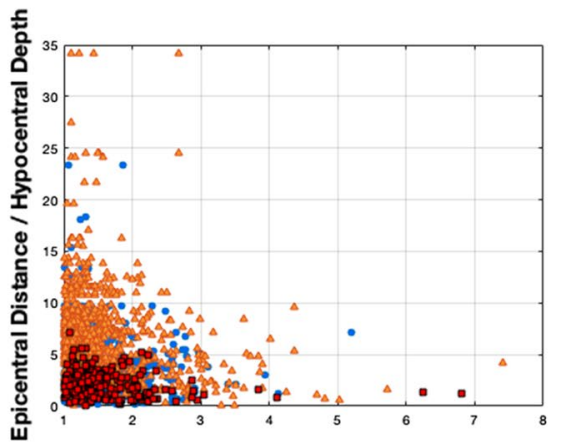

$\mathrm{Sa}(\mathrm{T}=1.0)$ As-recorded Larger Comp. / Smaller Comp.

Fig. 7 Comparison of the correlation of epicentral distance and the ratio of the epicentral distance to the hypocentral depth with the observed polarity

perpendicular-to-BAZ and rotated-to-max-PGA angles are not highly correlated in the dataset used in this paper. This is also the reason why variances of perpendicular-toBAZ and rotated-to-max-PGA are quite different in Fig. 5.

The relation of the distance with the polarity is another point of discussion when modelling the component-to-component variance. Although the GMM v5 componentto-component variance model by Bommer et al. (2018) specifically relates the polarity to distance (together with magnitude), it is shown in Fig. 7 that the distance has a 
weaker correlation with the polarity as compared to that of the distance-to-depth ratio for the records from outside Groningen. The depth of Groningen earthquakes is constantly $3 \mathrm{~km}$, thus the plots on the left and on the right in Fig. 7 have similar shape for the Groningen data (i.e. values in the y axis in the left are divided to a constant and presented on the right). This is not the case for the non-Groningen data as the depth varies in every event. Knowing that the radiation pattern is one of the most plausible explanations for the observed polarity in these small-magnitude earthquakes, it is expected that the distance-to-depth ratio helps to decrease the scatter in a component-to-component variance model for the case of events with varying depth.

\subsection{Implications of polarity in risk models and the proposed approach}

Risk models employ simplified structural representations, i.e. simple frames, fragility functions, mechanics based capacity equations or SDOF models. This is because the number of analyses required is excessive due to the need for including several possible variables in the model, such as different building typologies, ground motion parameters, soil parameters, etc. Component-to-component variability is one of the many of these parameters that need to be taken into account, and it becomes much more relevant if the structural typology in question has high strength and stiffness differences in orthogonal directions. An example is shown in Fig. 8 where a representative structural plan is placed on the polarity plot of the Groningen record. The representative structure has one weak and one strong structural direction. Figure $8 \mathrm{a}, \mathrm{b}$ show the cases where the weak and strong structural directions, respectively, perfectly coincide with the rotatedto-max-PGA direction of the motion. Figure $8 \mathrm{c}$ is the case where the rotated-to-maxPGA of the motion is in an arbitrary orientation in respect to the orthogonal structural axes. In order to better explain the dependence of the structural response to the ground motion orientation, the angle $\theta$ is shown in Fig. $8 \mathrm{~d}$ as the smaller angle (i.e. always between $0^{\circ}$ and $90^{\circ}$ ) between the weak structural direction and the rotated-to-max-PGA direction.

Variances from perpendicular-to-BAZ can be used as an alternative in non-ergodic hazard models, provided that the path non-ergodicity is available. In case an ergodic hazard model is used and high strength and stiffness differences are present in a certain structural typology, the risk model for that specific typology can take into account the difference in ground motion components in various ways, such as:

1. By using one of the two structural directions, depending on the purpose of the risk study, with a component-to-component variability coming from the records as-recorded (i.e. the use of weak direction in Crowley and Pinho 2017).

2. By repeating the analyses for both weak and strong structural directions with a component-to-component variability coming from the records as-recorded.

3. By running several orientation scenarios, i.e. with 15 degree intervals, where the rotatedto-max-PGA and the perpendicular directions of the motion coincide with several different angles of the structural model, as in Fig. 8c.

The last option described above has similarities with the GMRotDpp presented by Boore et al. (2006), that is "the $\mathrm{pp}^{\text {th" }}$ " percentile of the set of geometric means for a given oscillator's period. The approach proposed in this paper and further explained below, 


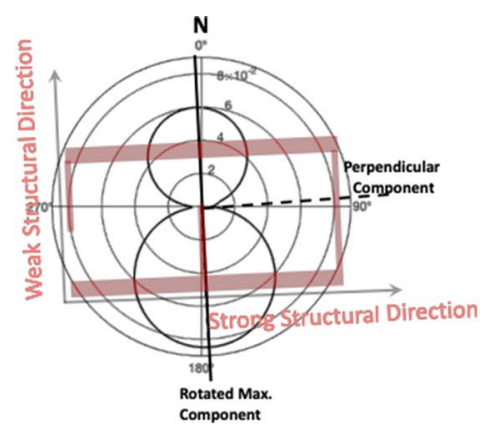

(a)

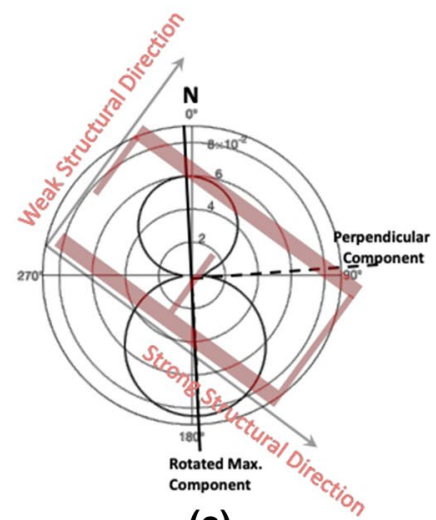

(c)
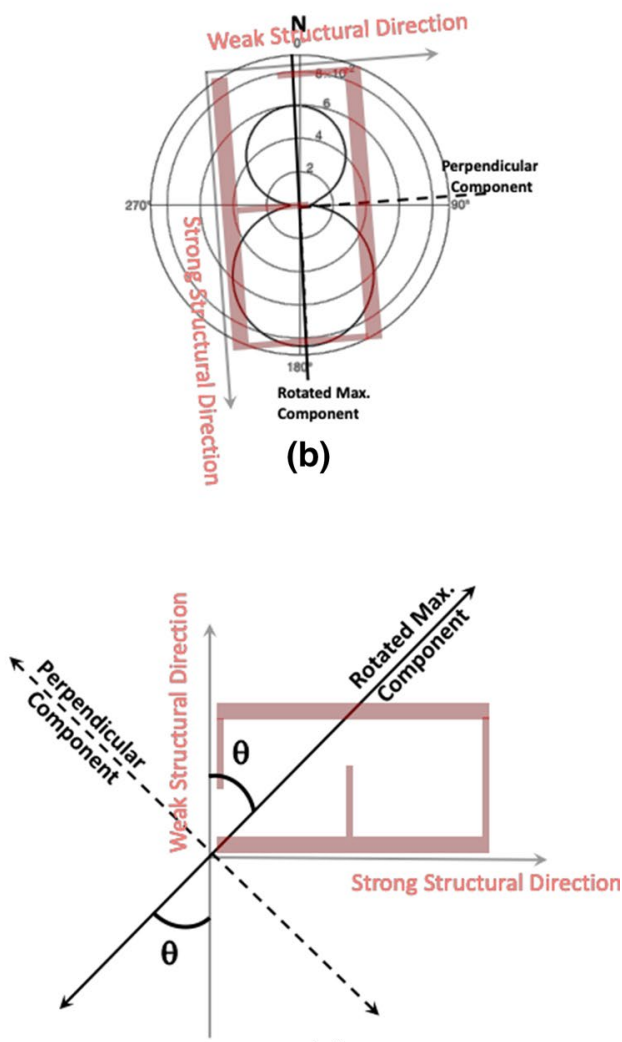

(d)

Fig. 8 Orientation of weak and strong structural directions in respect to the ground motion components, a coincidence of the weak structural direction with the component rotated-to-max-PGA, b coincidence of the strong structural direction with the component rotated-to-max-PGA, c structural directions arbitrarily oriented in respect to the ground motion components, and $\mathbf{d}$ structural plan of a building with significant differences of strength and stiffness in orthogonal plan direction, and definition of the $\theta$ angle

however, is based on the direction of the max PGA and not on different oscillator periods, because it directly assigns the rotated-to-max-PGA as the dominant motion, similar to the RotD100. In an answer to the evaluation panel, GMM v5 report (Bommer et al. 2018) discusses the relevance of RotD0 and RotD100 with the component-to-component variance and concludes that the variances would not be the same for RotD0 and RotD100 directions. This is confirmed by the exercise that has been run for this study and shown in Fig. 9. In that figure, it can be shown that all three datasets exhibit very low variances in case the instrument responses are rotated $45^{\circ}$ from the rotated-to-max-PGA direction. This position is where the two principal components are averaged trigonometrically, thus the variance goes down to its minimum. This is a response that would be similar to RotD0. On the other hand, the variances when the instrument responses are aligned with the rotated-to-maxPGA are very high, a result that would be similar to RotD100.

If one needs to take into account variations of structural orientations in respect to the ground motion, as explained in the last option above, that could be done by using a component-to-component variance model that takes into account the orientation angle of the 


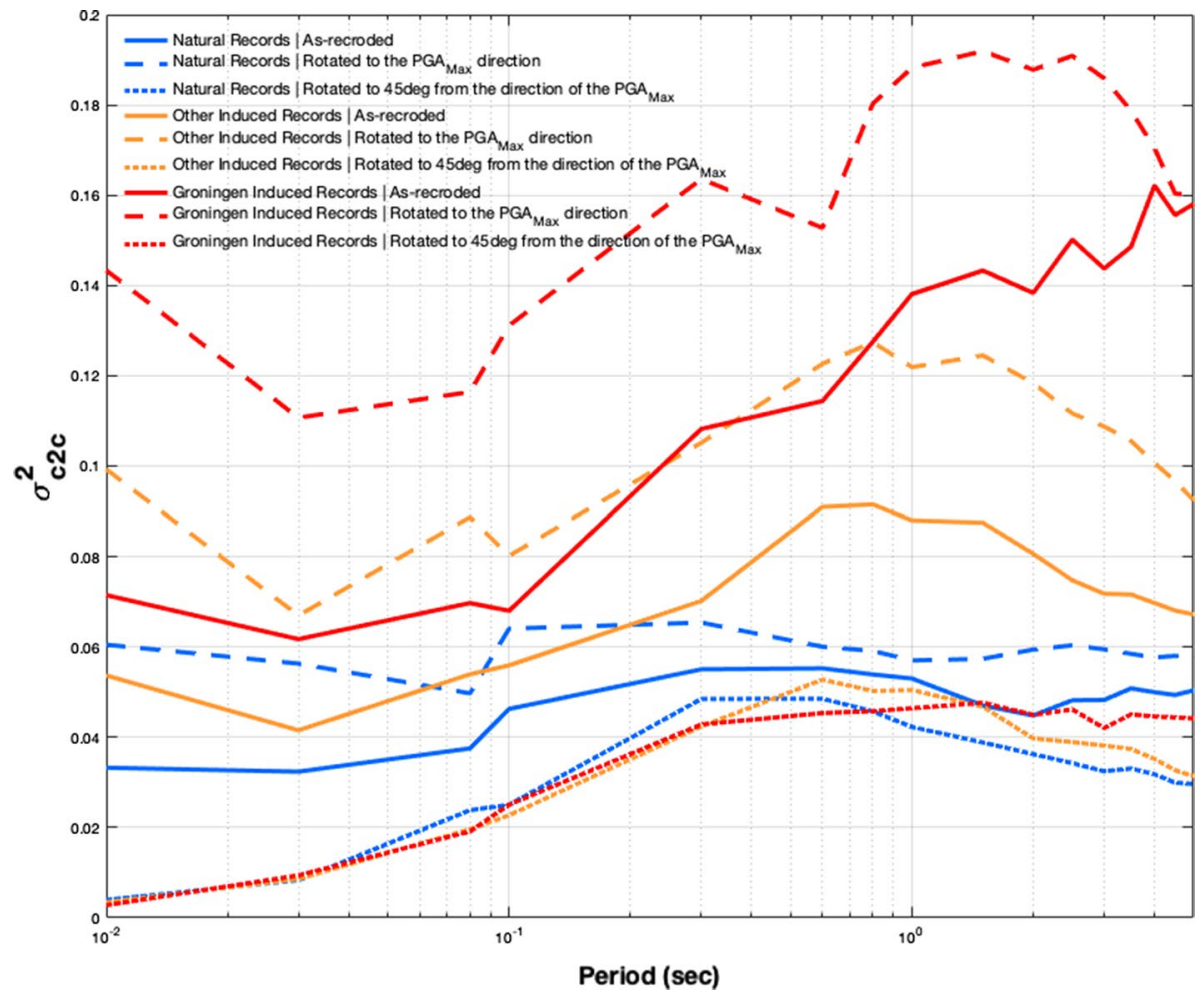

Fig. 9 Comparison of the component-to-component variances coming from the records as-recorded with the variances where the instrument response is aligned with the rotated-to-max-PGA angles and with a position $45^{\circ}$ from that, providing the maximum and the minimum variances for all three datasets

structure in respect to the rotated-to-max-PGA angle of the motion. Because this angle cannot be known before actually recording the motion, the variance model would thus be needed to take this into account in a probabilistic fashion. This can be done by assuming that the building orientation in respect to the strong motion components is not biased, i.e. the $\theta$ angle shown in Fig. $8 \mathrm{~d}$ is uniformly distributed between $0^{\circ}$ and $90^{\circ}$ for all structures. Please note that angles above $90^{\circ}$ would not matter as the spectral values are absolute maxima, without any sign.

In order to adapt the approach described above, the GMM v5 component-to-component variance model (Bommer et al. 2018) is used here, and a slight modification is proposed. The model (example curves for the model are shown in Fig. 4) consists of three regimes for three period ranges as given below in Eqs. 3-5.

$$
\begin{gathered}
\sigma_{c 2 c}^{2}(M, R)=0.026+1.03[5.6-\min (5.6, \max [M, 3.6])] R^{-2.22} \text { for } T \leq 0.10 \mathrm{~s} \\
\sigma_{c 2 c}^{2}(M, R)=0.045+5.315[5.6-\min (5.6, \max [M, 3.6])] R^{-2.92} \text { for } T \geq 0.85 \mathrm{~s} \\
\sigma_{c 2 c}^{2}(T, M, R)=\sigma_{c 2 c}^{2}(0.1, M, R)+\left[\frac{\log (T)-\log (0.1)}{\log (0.85)-\log (0.1)}\right] \\
{\left[\sigma_{c 2 c}^{2}(0.85, M, R)-\sigma_{c 2 c}^{2}(0.10, M, R)\right] \text { for } 0.1 \mathrm{~s}<T<0.85 \mathrm{~s}}
\end{gathered}
$$



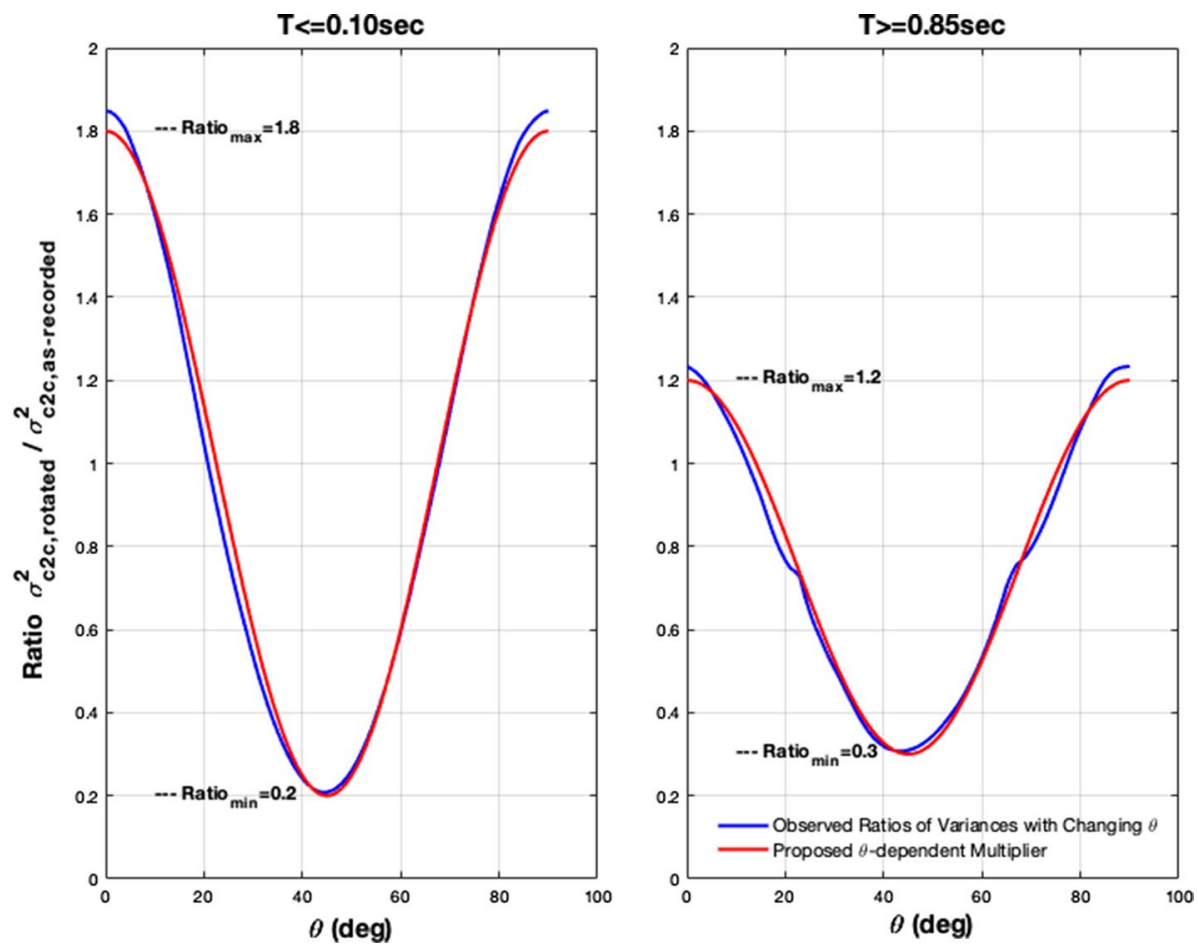

Fig. 10 Ratio of the component-to-component variances as-recorded to rotated, where all records are individually rotated to their rotated-to-max-PGA angle, and then rotated back (i.e. deviated) $1^{\circ}$ at a time until $90^{\circ}$ rotation is achieved

As can be seen in the examples in Fig. 4, the Bommer et al. (2018) model has two straight lines in respect to the period, one given by Eq. 3 and the other with Eq. 4 . When the component-to-component variances are calculated for the three datasets for the records as-recorded, and compared to those calculated by using the rotated-to-max-PGA angle and a position $45^{\circ}$ from that, an envelope of maximum and minimum variances is obtained, as shown in Fig. 9. Apart from $0^{\circ}$ and $45^{\circ}$ from the rotated-to-max-PGA, other angles have also been used, and the ratios of the individually rotated variances to the as-recorded variances have been calculated for $\theta$ angles between $0^{\circ}$ and $90^{\circ}$. The ratios of the rotated variances to the as-recorded variances have been calculated for the two straight parts of the Bommer et al. (2018) variance model, once for the short period range $(\mathrm{T}<0.1 \mathrm{~s})$ and once for the long-period range $(\mathrm{T}>0.85 \mathrm{~s})$. These two results can be seen in Fig. 10. The ratio of the variances of rotated to as-recorded motions follows a cosine-like curve in respect to the rotation angle $\theta$. A cosine model has been fit into the observation, as shown in Eq. 6:

$$
\Gamma(\theta)=\frac{\text { Ratio }_{\text {max }}-\text { Ratio }_{\text {min }}}{2} \cos (4 \theta)+\frac{\text { Ratio }_{\text {max }}+\text { Ratio }_{\text {min }}}{2}
$$

Here the Ratio ${ }_{\max }$ and Ratio $_{\min }$ are the minimum and maximum ratios of the observed variances of rotated to as-recorded, as shown in Fig. 10. These values are obtained as 0.20 and 1.80 for short periods and as 0.3 and 1.20 for the long periods. These are linear 
multipliers for the straight parts of the Bommer et al. (2018) model, thus the orientationdependent variance model is proposed in Eqs. 7 and 8 below. The inclined part of the curves, given in Eq. 5, can remain the same as it geometrically connects the two straight lines that are period-independent.

$$
\begin{array}{cc}
\sigma_{c 2 c}^{2}(\theta, M, R)=0.026 \Gamma+1.03[5.6-\min (5.6, \max [M, 3.6])] R^{-2.22} & \text { for } T \leq 0.10 \mathrm{~s} \\
\sigma_{c 2 c}^{2}(\theta, M, R)=0.045 \Gamma+5.315[5.6-\min (5.6, \max [M, 3.6])] R^{-2.92} & \text { for } T \geq 0.85 \mathrm{~s}
\end{array}
$$

The approach proposed above allows the user to pick up several $\theta$ angles in the cosine model (Eq. 6 and Fig. 10) with equal probabilities and alter the component-to-component variance model each time. The components derived from the geometric-mean GMM spectrum can then be applied to specific structural directions individually, because the $\theta$ angle relates the structural axes to the ground motion directions.

\section{Discussion and conclusions}

This paper scrutinizes the issue of high polarity previously reported to be discriminating aspect of the Groningen earthquake records. The purpose of the study is to investigate how Groningen earthquake records exhibit strong component-to-component differences as compared to other induced seismic events and to natural events, and to discuss whether these differences can have implications on the risk models. The paper concludes with a proposal of an approach to take into account the structural orientation in respect to the ground motion directions. In order to achieve this, a comparative dataset has been created with natural seismic events that have similar characteristics in terms of magnitude, epicentral distances, depth and soil properties of the recording stations. A total of 1831 recording suites (i.e. couples of horizontal components) are used. 202 of these are Groningen records, 1112 are from other induced seismicity events with geothermal, waste water injection and hydraulic fracturing activities, while 517 are from natural earthquakes. It should be noted that the magnitude and distance of the Groningen records are in a very small range, a fact that renders finding similar earthquake records rather difficult. Groningen records used here come from earthquakes with magnitudes from 2 to 3.6 and distances up to $22 \mathrm{~km}$. The depth is constantly $3 \mathrm{~km}$.

Component-to-component variances are calculated for periods between 0.01 and $5 \mathrm{~s}$, and presented for Groningen data as well as for other induced and natural records. The variances are compared to previously reported tectonic variance models as well as to the Groningen-specific variance model. It is shown that the variances for other datasets decrease after $0.6 \mathrm{~s}$ period while they constantly increase for the Groningen records. In other words, the high component-to-component variance persists even in long periods in the Groningen data, as opposed to the rest of the data used in comparison.

Sensitivity of the component-to-component variance to instrument orientation has also been investigated by rotating all sensors with 1-degree intervals (some of the results are presented in 15-degree intervals). It was found that the range of changes in the variances remains in the order of $15 \%$ for the case of Groningen. This is close to what was already reported in the Groningen GMM v5. However, it is also argued in the presented paper that the low variation in variances is rather expected in this case because all records are rotated to the same arbitrary angle simultaneously, averaging thus component-to-component 
variances throughout the entire field. This is reason why two other rotations have also been exercised here, which are rotated-to-max-PGA and perpendicular-to-BAZ. The former is the direction of the rotated component with the highest PGA, identified by rotating the two horizontal components simultaneously by a small angle at a time, while the latter is the direction perpendicular to the station back-azimuth. The perpendicular-to-BAZ is used for marking the theoretical shear wave direction that occurs perpendicular to the travel path. It was shown that the rotated-to-max-PGA provides the highest variances, increasing variances for all datasets up to 2 times in short periods. The direction perpendicular-to-BAZ, on the other hand, does not provide much difference in the variances as compared to the use of as-recorded components.

The relation of the distance with the polarity has also been investigated. It is shown that the distance has a weaker correlation with the polarity as compared to that of the distanceto-depth ratio for the records from outside Groningen. The depth of Groningen earthquakes is constantly $3 \mathrm{~km}$, thus the correlations between the polarity and depth or distance-todept ratio are practically the same. Knowing that the radiation pattern is one of the most plausible explanations for the observed polarity in these small-magnitude earthquakes, it is expected however that the distance-to-depth ratio helps in decreasing the scatter in a component-to-component variance model for the case of events with varying depth.

An angle, $\theta$, that is the smaller angle (i.e. always between $0^{\circ}$ and $90^{\circ}$ ) between the weak direction of the structure and the rotated-to-max-PGA direction of the motion is defined to better explain the relevance of polarity with the structural response. It was shown that when $\theta$ is between $0^{\circ}$ and $45^{\circ}$, the variances exhibit their maximum and minimum values, enveloping the variances of all other $\theta$ angles. The ratios of the variances from the rotated components to the variances from the as-recorded components have been plotted for short and long periods, and it was shown that this ratio fluctuates in a cosine form, varying between 0.2 and 1.8 for short periods $(\mathrm{T}<0.1 \mathrm{~s}$ ) and between 0.3 and $1.2 \mathrm{~s}$ for long periods $(\mathrm{T}>0.85 \mathrm{~s}$ ). Based on that finding, a modification to the Groningen GMM v5 componentto-component variance model is proposed, where the $\theta$ angle is used as one of the parameters of the function. Authors could only discuss the possible uses of this approach theoretically in this paper, without providing an exercise, but this issue will be further investigated in future studies.

\section{Data and resources}

The Oklahoma data is given by Dr. Abhineet Gupta, and details of that data can be found in Gupta et al. (2017). The Groningen data was downloaded and processed by using the Python based ObsPy code (Beyreuther et al. 2010). Turkish data was manually downloaded from deprem.afad.gov.tr (last accessed 17th of October 2018). Greek seismic data was downloaded from EUROSEIS website that is maintained by the Unit of Soil Dynamics and Geotechnical Earthquake Engineering, Aristotle University of Thessaloniki (1993): EUROSEISTEST Strong Motion Network. https://doi.org/10.7914/SN/EG (Pitilakis et al. 2013). The rest of the data was located and downloaded by using IRIS services. The facilities of IRIS Data Services, and specifically the IRIS Data Management Center, were used for access to waveforms, related metadata, and/or derived products used in this study. IRIS Data Services are funded through the Seismological Facilities for the Advancement of 
Geoscience and EarthScope (SAGE) Proposal of the National Science Foundation under Cooperative Agreement EAR-1261681.

A flat file providing details of all the records used can be downloaded from https://githu b.com/ihsanenginbal/Bal-etal.-2018-BEE-Paper.git.

Acknowledgements The Oklahoma dataset and some of the scripts for processing that data were generously provided by the Resilience Engineering Lead at One Concern, Inc., Dr. Abhineet Gupta. The authors are grateful to his contribution. The authors would like to thank Julian Bommer for the valuable discussion he provided when an earlier version of the work was presented in the GMPE v5 workshop. The authors are grateful to Prof. Bozidar Stojadinovic and Catherine Whyte for helping to find some of the Swiss and US induced seismicity records. Bernard Dost and Elmer Ruigrok from KNMI were helpful in identifying and excluding the problematic data, while Jordi Domingo Ballesta and Janneke van Ginkel from KNMI were helpful in accessing the public KNMI data. Their help is noted with gratitude. A note of gratitude is also due to Gail Atkinson for helping to use the Alberta induced seismicity catalogue. We also gratefully acknowledge the great deal of help by Ahmet Anıl Dindar in using Python codes for processing the seismic data.

\section{References}

Abrahamson NA, Silva WJ, Kamai R (2014) Summary of the Abrahamson, Silva, and Kamai NGA-West2 ground-motion relations for active crustal regions. Earthq Spectra 30:1025-1056

Atkinson GM (2015) Ground-motion prediction equation for small-to-moderate events at short hypocentral distances, with application to induced-seismicity hazards. Bull Seismol Soc Am 105:981-992. https:// doi.org/10.1785/0120140142

Atkinson GM, Assatourians K (2017) Are ground-motion models derived from natural events applicable to the estimation of expected motions for induced earthquakes? Seismol Res Lett 88:430-441. https://doi. org/10.1785/0220160153

Atkinson GM, Wald D, Worden CB, Quitoriano V (2018) The intensity signature of induced seismicity. Bull Seismol Soc Am 108:1-7. https://doi.org/10.1785/0120170316

Beyer K, Bommer JJ (2007) Selection and scaling of real accelerograms for bi-directional loading: a review of current practice and code provisions. J Earthq Eng 11:13-45

Beyreuther M, Barsch R, Krischer L, Megies T, Behr Y, Wassermann J (2010) ObsPy: a Python toolbox for seismology. SRL 81(3):530-533. https://doi.org/10.1785/gssrl.81.3.530

Bommer J, Dost B, Edwards B, Kruiver PP, Meijers P, Ntinalexis M, Rodriguez Marek A, Ruigrok E, Spetzler J, Stafford P (2017a) V4 ground-motion model (GMM) for response spectral accelerations, peak ground velocity and significant duration in the Groningen field. Report prepared for NAM

Bommer JJ, Stafford PJ, Edwards B et al (2017b) Framework for a ground-motion model for induced seismic hazard and risk analysis in the Groningen gas field, the Netherlands. Earthq Spectra 33:481-498. https://doi.org/10.1193/082916EQS138M

Bommer J, Edwards B, Kruiver PP, Rodriguez-Marek A, Stafford PJ, Dost B, Ntinalexis M, Ruigrok E, Spetzler J (2018) V5 ground-motion model for the Groningen field. Report prepared for NAM

Boore DM (2005) Erratum: Equations for estimating horizontal response spectra and peak acceleration from western North American earthquakes: a summary of recent work, by D.M. Boore, W.B. Joyner and T.E. Fumal. Seismol Res Lett 76(3):368-369

Boore DM, Watson-Lamprey J, Abrahamson NA (2006) Orientation-independent measures of ground motion. Bull Seismol Soc Am 94(4A):1502-1511

Bourne SJ, Oates SJ, Bommer JJ et al (2015) A monte carlo method for probabilistic hazard assessment of induced seismicity due to conventional natural gas production. Bull Seismol Soc Am 105:1721-1738. https://doi.org/10.1785/0120140302

Boyd OS, McNamara DE, Hartzell S, Choy G (2017) Influence of lithostatic stress on earthquake stress drops in North America. Bull Seismol Soc Am 107:856-868. https://doi.org/10.1785/0120160219

Campbell KW, Bozorgnia Y (2007) Campbell-Bozorgnia NGA ground motion relations for the geometric mean horizontal component of peak and spectral ground motion parameters. Pacific Earthquake Engineering Research Center, Berkeley

Crowley H, Pinho R (2017) Report on the v5 fragility and consequence models for the Groningen field. Report for NAM 
Crowley H, Polidoro B, Pinho R, van Elk J (2017) Framework for developing fragility and consequence models for local personal risk. Earthq Spectra 33:1325-1345. https://doi.org/10.1193/083116EQS1 $40 \mathrm{M}$

Dost B, van Eck T, Haak H (2004) Scaling of peak ground acceleration and peak ground velocity recorded in the Netherlands. Bull Geofis Teor Appl 45(3):153-168

Goertz-Allmann BP, Goertz A, Wiemer S (2011) Stress drop variations of induced earthquakes at the Basel geothermal site. Geophys Res Lett. https://doi.org/10.1029/2011g1047498

Gupta A, Baker JW, Ellsworth WL (2017) Assessing ground-motion amplitudes and attenuation for smallto-moderate induced and tectonic earthquakes in the central and eastern United States. Seismol Res Lett 88(5):1379-1389. https://doi.org/10.1785/0220160199

Hough SE (2014) Shaking from injection-induced earthquakes in the central and eastern United States. Bull Seismol Soc Am 104:2619-2626. https://doi.org/10.1785/0120140099

Kaski KM, Atkinson GM (2017) A comparison of ground-motion characteristics from induced seismic events in Alberta with those in Oklahoma. Seismol Res Lett 88:1570-1585. https://doi. org/10.1785/0220170064

Kraaijpoel D, Dost B (2012) Implications of salt-related propagation and mode conversion effects on the analysis of induced seismicity. J Seismol 17:95-107. https://doi.org/10.1007/s10950-012-9309-4

Liu J, Lin J, Alatas A et al (2016) Seismic parameters of hcp-Fe alloyed with Ni and Si in the Earth's inner core. J Geophys Res Solid Earth 121:6597-6607. https://doi.org/10.1002/2015jb012625.received

Pitilakis K, Roumelioti Z, Raptakis D, Manakou M, Liakakis K, Anastasiadis A, Pitilakis D (2013) The EUROSEISTEST strong ground motion database and web portal. Seismol Res Lett 84(5):796-804

Smyrou E, Tasiopoulou P, Bal IE, Gazetas G (2011) Ground motions versus geotechnical and structural damage in the February 2011 Christchurch earthquake. Seismol Res Lett 82:882-892. https://doi. org/10.1785/gssrl.82.6.882

Stafford PJ, Zurek BD, Ntinalexis M, Bommer J (2018) Extensions to the Groningen ground-motion model for seismic risk calculations: component-to-component variability and spatial correlation. Bull Earthq Eng. https://doi.org/10.1007/s10518-018-0425-6

Sumy DF, Neighbors CJ, Cochran ES, Keranen KM (2017) Low stress drops observed for aftershocks of the 2011 Mw5.7 Prague, Oklahoma, earthquake. J Geophys Res Solid Earth 122:3813-3834. https://doi. org/10.1002/2016JB013153

Wu Q, Chapman M, Chen X (2018) Stress-drop variations of induced earthquakes in Oklahoma. Bull Seismol Soc Am 108:1107-1123. https://doi.org/10.1785/0120170335

Yenier E, Atkinson GM (2015) Regionally adjustable generic ground-motion prediction equation based on equivalent point-source simulations: application to central and eastern North America. Bull Seismol Soc Am 105:1989-2009. https://doi.org/10.1785/0120140332

Yenier E, Atkinson GM, Sumy DF (2017) Ground motions for induced earthquakes in Oklahoma. Bull Seismol Soc Am 107:198-215. https://doi.org/10.1785/0120160114

Zaliapin I, Ben-Zion Y (2016) Discriminating characteristics of tectonic and human-induced seismicity. Bull Seismol Soc Am 106:846-859. https://doi.org/10.1785/0120150211

\section{Affiliations}

\section{İhsan Engin Bal ${ }^{1}$ [ $\cdot$ Eleni Smyrou ${ }^{1} \cdot$ Dimitris Dais $^{1}$}

İhsan Engin Bal

i.e.bal@pl.hanze.nl

1 Research Centre for Built Environment NoorderRuimte, Hanze University of Applied Sciences, Zernikeplein 11,9701 DA Groningen, The Netherlands 